\title{
GenPolis - Prototipagem e aplicação de uma ferramenta especializada para otimização via algoritmos genéticos de planos fixos de sinalização semafórica em sub-redes urbanas
}

\author{
${ }^{1}$ Bruno Sarno Mugnela, ${ }^{1}$ Marcio Lobo Netto \\ ${ }^{1}$ Departamento de Engenharia de Sistemas Eletrônicos (PSI) \\ Escola Politécnica da Universidade de São Paulo (EPUSP) \\ CEP 05508-010 - São Paulo - SP - Brasil \\ bruno.mugnela@usp.br, marcio.netto@poli.usp.br
}

\begin{abstract}
There is a considerable knowledge base for traffic engineering optimization using genetic algorithms, but rare are the real applications on environments that lack on traffic flow data or Intelligent Transportation Systems (ITS). This paper summarizes a new simulation model over which a genetic algorithm is applied for finding signal timing plans that reduce stops and delays in congested urban sub-networks. The tool is simplified for achieving fast running times using only the parameters measured by the Traffic Engineering Company of the city of São Paulo (CET-SP). The case study in one of its sub-networks resulted in approximate $30 \%$ reduction of the delays registered in the simulations of the previous plans.
\end{abstract}

Resumo. A aplicação de algoritmos genéticos (AGs) para otimizações em engenharia de tráfego possui boa base bibliográfica, mas são raras as aplicações reais em meios onde há escassez de dados e de sistemas inteligentes de controle de tráfego. Este artigo apresenta um novo modelo de simulação sobre o qual um AG é aplicado para encontrar planos de sinalização semafórica que reduzam atrasos e paradas em sub-redes congestionadas. A ferramenta é simplificada para execução rápida, usando apenas os parâmetros colhidos pela CET-SP. O estudo de caso em uma subrede paulistana resultou em reduções da ordem de 30\% no nível de atraso calculado nas simulações dos planos anteriores.

\section{Introdução}

Para a solução dos problemas de congestionamento em grandes cidades, as ações são tanto no sentido de aumentar a infraestrutura viária e melhorar o seu funcionamento com obras de expansão e reformas, quanto de controlar a frota de veículos por meio de regulamentações e ajustes de sinalização. Em Lopes (2003) são descritos exemplos relevantes desses tipos de iniciativa. Nos últimos anos testemunhamos a implantação de algumas ferramentas de suporte ao controle de tráfego, como câmeras de monitoramento, radares para aferição de velocidade média, sensores como laços e controle semafórico ativo por meio de sistemas centralizados dotados de métodos como o SCOOT [Robertson e Bretherton, 1991]. Em cidades como São Paulo obstáculos variados impedem que estes recursos estejam plenamente disponíveis ou, quando disponíveis, facilmente acessíveis e manipuláveis para os operadores de tráfego. Ainda 
em Lopes (2003), são levantados pontos importantes relativos às limitações dessas medidas como escassez de recursos financeiros e impactos reduzidos a longo prazo, pois o comportamento da frota se altera constantemente. Neste sentido, uma vez que melhorias aumentam a capacidade de determinada região urbana, esta passa a atrair mais usuários, criando uma nova situação de equilíbrio na rede. Assim vemos que algo que se pode fazer de forma mais ágil para adaptar a resposta das malhas viárias é o ajuste frequente dos planos de sincronização semafórica fixa.

Para a temporização de uma rede sincronizada de semáforos existem três parâmetros principais a serem configurados, tempo de ciclo, tempos de verde, e as defasagens que determinam o instante de início de funcionamento entre os ciclos de cada semáforo (em relação a um semáforo de referência). Para planos de temporização fixa, quando se leva em conta apenas o comportamento de uma única intersecção, a determinação dos dois primeiros parâmetros envolve um processo relativamente simples. Mas, mesmo esse procedimento faz uso de dados cuja atribuição é baseada na experiência (ajuste e observação) dos órgãos operadores de tráfego [Vilanova, 2005a].

Quando a questão envolve o funcionamento de vários semáforos em uma rede sincronizada essa tarefa fica mais difícil, e uma importante parte do trabalho reside em se determinar o conjunto correto de defasagens entre os diferentes semáforos. No caso de malhas viárias, métodos analíticos não representam a melhor forma para analisar a interação entre os elementos da malha viária, e ao mesmo tempo, envolvem explosões combinatórias que criam vastos espaços de buscas que não permitem uma procura exaustiva pela solução ótima global [Lebdeh e Benekohal, 1997].

Em função da característica mencionada, os métodos clássicos de coordenação semafórica em rede, como o do TRANSYT-7F [Hale, 2008], não utilizam algoritmos de otimização convencionais e não representam a interação de forma analítica, preferindo usar métodos de simulação de tráfego. Nessas ferramentas, métodos de busca de ótimos locais foram aplicados de forma complementar, ajustando a sinalização a partir de configurações baseadas nas observações dos engenheiros de tráfego. Um exemplo desses métodos é o hill-climbing, formulado com o compromisso de encontrar o ótimo local pertencente à região do plano de busca que compreende a solução inicial dada como entrada. Mais recentemente, meta-heurísticas de procura global foram propostas e vêm sendo usadas com sucesso para otimização de sinalização e outros problemas de engenharia que envolvam explosões combinatórias. Para uma descrição formal de muitos deles, recomenda-se Brownlee (2011).

Este trabalho tem então por objeto propor uma ferramenta dotada de uma heurística para realizar otimizações de temporização fixa de sub-redes de semáforos, de modo a contribuir para a melhoria do fluxo de tráfego em sub redes viárias de grandes centros urbanos. Tal proposta incorpora tarefas de otimização de sinalização semafórica através de Algoritmos Genéticos (AGs) [Mitchell, 1995]. Esse método é acoplado a um simulador de tráfego simplificado e rápido que usa, para estabelecer o comportamento do trânsito, apenas os parâmetros que provenientes de estudos para revisão de planos semafóricos na cidade São Paulo [Ejzenberg, 2005; Vilanova, 2005b] cuja malha viária contém a sub-rede usada no estudo de caso retratado ao fim deste artigo. Logo, neste trabalho pretende-se apresentar um panorama experimental acerca da aplicabilidade de AGs para a realização de melhorias no trânsito de cidades como São Paulo. 


\section{Estratégias Evolucionárias e Algoritmos Genéticos}

Seja qual for a forma que qualquer espécie de ser vivo tenha adquirido com a evolução, ela decorre de uma adaptação para sobrevivência, sendo que nesse processo não há restrições acerca de como as soluções podem se apresentar e o caminho que devem tomar. Foi a partir desse princípio dos processos evolutivos, dos inúmeros caminhos possíveis que podem ser tomados até a emergência de uma solução, que se pensou na hipótese da aplicação desses conceitos para resolver problemas da vida prática. E com isso surgiram os programas que são classificados pelo nome desta seção. Para resumir o resultado da inspiração nos mecanismos naturais de evolução cabe começar por enumerar os passos de um procedimento comum na área de AGs [Rennard, 2000]:

Inicialização:

1. Codificação do problema, normalmente em uma série binária (cromossomo);

2. Criação aleatória (ou não) de uma população inicial de soluções/cromossomos;

Ciclo evolutivo:

3. Atribuição de uma pontuação (fitness, ou índice de mérito) para cada solução;

4. Seleção de soluções de acordo com seus níveis de adequação;

5. Recombinações entre os cromossomos selecionados e criação de nova geração.

Geralmente o espaço amostral que compreende as alternativas para um problema complexo é muito grande, o que torna complicada a identificação de um traçado ao redor das regiões que contenham as melhores soluções. O AG é capaz de contornar esse problema começando com a geração de uma população inicial de vários indivíduos que disparam uma exploração paralela de diferentes regiões. Mas o que confere ao AG sua eficácia é o seu paralelismo implícito, explicado nos próximos parágrafos.

É comum fazermos a descrição de um indivíduo por partes, de modo que ao descrevermos vários indivíduos, muitas vezes nos deparamos com eventuais semelhanças entre eles. Desta forma alguém que, por exemplo, tenha braços longos representa inevitavelmente a população dos indivíduos que também possuem este traço. Trazendo essa premissa para o AG, na codificação de um problema a partir de palavras de oito bits, teremos então que, assim como um braço, cada conjunto de dois ou mais bits constitui um bloco de construção da solução, sendo que no espaço de busca, cada bloco representa uma região distinta (por exemplo: $10 * * * * * * ; * * 00 * * * * *$ ).

Assim podemos perceber que existe um paralelismo implícito na exploração do espaço amostral, na medida em que um único cromossomo representa diversas regiões ou populações de outros cromossomos ao mesmo tempo. Por sua vez, os blocos de construção específicos que têm efeito importante na adequação do indivíduo ao problema possuem chances maiores de se propagar por diversas gerações e permitir a exploração de boas regiões. Por fim a propagação e união desses blocos através dos cruzamentos é o que permite a rápida convergência de um $\mathrm{AG}$ a bons resultados.

Importante notar que os tempos de convergência são muito dependentes dos parâmetros de execução. Uma avaliação introdutória na área pode ser encontrada em Cavicchio (1972), onde são medidos os tempos de convergência para diferentes conjuntos de parâmetros e o nível de melhora trazido pelos mesmos. 


\section{AGs Aplicados à Engenharia de Tráfego}

Nessa seção é apresentada uma revisão referenciando alguns trabalhos importantes que envolveram estudos de AGs em controle de tráfego ao longo dos últimos quinze anos.

Em Lebdeh e Benekohal (1997) o foco da aplicação é a prevenção da formação de bloqueios em que um sinal verde não libera carros devido ao enchimento do segmento à frente (jusante). Neste trabalho é indicada a necessidade de controle semafórico dinâmico para situações saturadas, pois o comportamento do tráfego em uma janela de tempo depende do que foi registrado na janela de tempo anterior. Além disso, o autor relata que execuções de AGs para condições iniciais dentro de certa faixa de dispersão (sementes de números aleatórios distintos) convergem a valores semelhantes.

No estudo realizado por Lo et al. (1999), um AG otimiza o tráfego simulando janelas de 45 minutos. Nele é indicado que em situações não congestionadas o recomendável seria otimizar um semáforo de cada vez, de forma serial, enquanto que em situações congestionadas seria mais interessante otimizar todos os semáforos paralelamente, sendo que ambas as estratégias apresentaram resultados melhores que aqueles alcançados pelo software Transyt-7F.

Para avaliar o impacto do uso de diferentes funções objetivo, Park et al. (2000) mediram o desempenho do AG em situações de saturação, e ao classificar os cromossomos de acordo com diversos critérios, chegaram ao indício de que o critério mais simples, o do atraso, é o mais eficiente. Outro trabalho interessante realizado por Park et al. (2004) envolveu o uso de AGs para a otimização de diversos planos ao longo de um dia de operação, e inclusive dos pontos de transição entre eles.

Ceylan e Bell (2004a) contemplaram o efeito dinâmico de longo prazo do comportamento da rede ao adicionarem ao AG um procedimento periódico em que o comportamento do tráfego simulado frente aos melhores planos encontrados é reavaliado, de forma a estabelecer a nova situação de equilíbrio entre oferta e demanda e assim remodelar as matrizes de origem-destino antes de prosseguir com a otimização. Em trabalho semelhante naquele ano [Ceylan e Bell, 2004b], os pesquisadores aferiram o aumento de capacidade da rede causado pelo mesmo método.

Cantarella et al. (2006) compararam, frente a tempos fixos de processamento, diversos métodos heurísticos de otimização, a saber o Hill Climbing, Tabu Search [Brownlee, 2011], um AG, e métodos híbridos. O autor identificou que os métodos AG e Tabu Search puros são os que convergem mais rapidamente, e constatou que o Hill Climbing foi o único que obteve performance consideravelmente pior, mas alertando que pode ao mesmo tempo ser o melhor se os outros forem configurados de maneira errada.

Também para contemplar as mudanças nos fluxos de tráfego, dois trabalhos, de Sun e Benekohal (2006) e Teklu et al. (2007) aplicaram ciclos simultâneos de otimização e reavaliação de fluxos, usando diferentes AGs, com os primeiros indicando que é necessário realizar diversas otimizações e os últimos que muito ainda deve ser feito para que se saiba selecionar os melhores parâmetros do AG.

Para aumentar a rapidez de execução do AG, Stevanovic et al. (2007), executaram um AG usando computação distribuída, e usaram como índice de mérito 
uma composição de atrasos e paradas (o total dos atrasos da rede em horas é somado ao número de paradas multiplicado por um fator). Com maior agilidade puderam avaliar diferentes conjuntos de parâmetros para execução do AG recomendando então o uso de números de gerações no mínimo três vezes maior que o tamanho da população, probabilidade de cruzamento de 40 a $70 \%$ e de mutação de 1 a $4 \%$.

Na mesma busca por boas configurações, Kesur (2009) avaliou outros elementos como os tipos de codificação, cruzamento e mutação, chegando aos indicativos de que o AG funciona melhor com o uso de código gray para uniformizar as mutações binárias. Além disso, em detrimento do método de corte simples, os resultados favoreceram o uso do cruzamento misturado, em que os cromossomos dos pais são quebrados em diversos pedaços, cada um contendo um parâmetro, com os filhos recebendo esses parâmetros de um ou outro pai de forma aleatória.

Em Medina et al. (2010) foi realizado estudo considerando diversos níveis de situações saturadas e não saturadas, levando à constatação de que o AG traz maiores ganhos em redes carregadas.

Em um trabalho interessante, Pohlmann e Friedrich (2010) ajudam a quebrar a barreira do AG como aplicação apenas offline e combinam uma ferramenta estimadora de tráfego a sistemas de monitoramento em tempo real e um modelo rápido de simulação por transmissão celular para desenvolver uma aplicação em pipeline que otimiza a sinalização semafórica a cada quinze minutos. A ferramenta foi aplicada em uma rede real com bons resultados, mas indicando que o estimador ainda apresenta problemas na hora de lidar com o tráfego mais volátil dos períodos de pico.

\section{A Ferramenta GenPolis}

O GenPolis é um simulador de trânsito mesoscópico de demanda dinâmica, concebido para simular rapidamente malhas complexas de vias concorrentes e semaforizadas. Nele, o simulador apresenta uma foto virtual do estado da rede para cada segundo simulado. $\mathrm{O}$ projeto possui um construtor preliminar responsável por transformar a malha viária, extraída de um mapa através de dados geográficos do website OpenStreetMap, automaticamente em um grafo conexo que é então tratado no programa CityEngine. O resultado é a obtenção rápida de redes geometricamente semelhantes às redes reais (Figura 1).
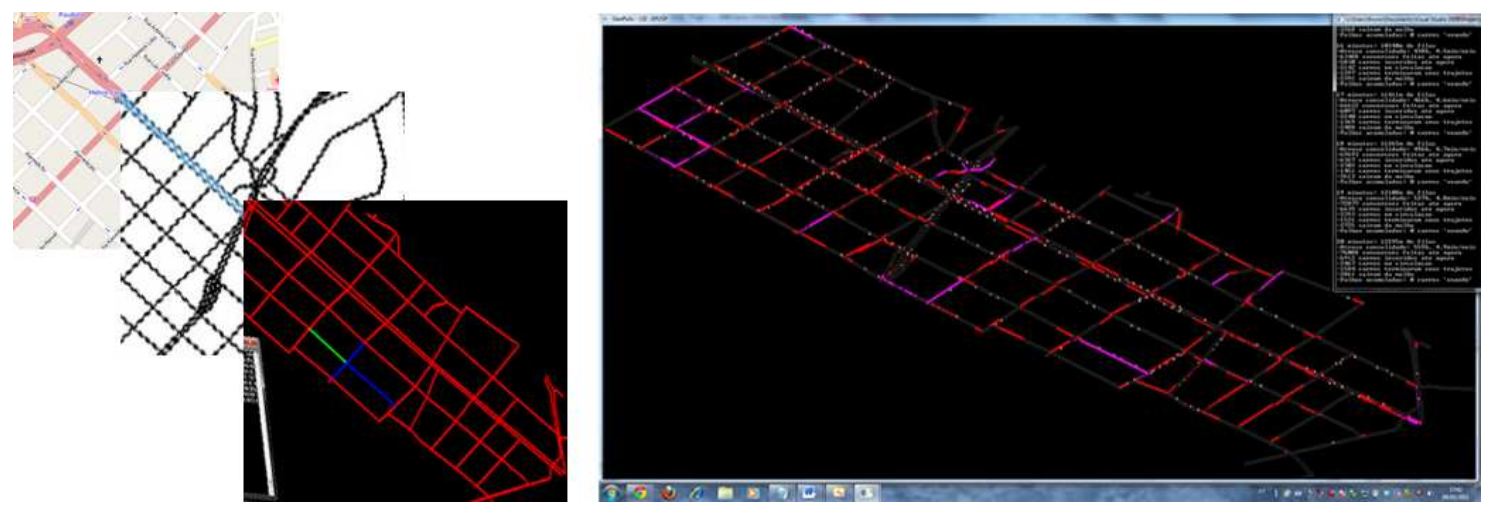

Figura 1. Processo de obtenção de uma rede de teste (região da Av.Paulista) 
O ambiente de simulação é composto basicamente de três entidades: o segmento de rua (Link), que se assemelha a uma esteira, movimentando todos os carros com igual velocidade do começo ao fim do segmento, onde são empilhados (o que apontaria para um modelo de fila vertical, como em versões antigas do Transyt, mas não no caso do GenPolis, onde há cálculo de fila horizontal, mais condizente com a realidade); o cruzamento (Nó), que pode conter um semáforo ou não e é responsável por distribuir os carros dos segmentos à montante para os segmentos conectados à jusante; e o carro, a partícula básica de carga do sistema.

Do lado da simulação microscópica temos apenas que o simulador descreve seus carros de forma unitária, enquanto que pelo lado macroscópico predominante, o comportamento dos carros é regido por determinações agregadas de velocidade fixa (em função do tempo de percurso medido em campo) e as conversões são baseadas em probabilidade. Logo os fluxos das malhas virtuais são compostos de forma estatística e consolidada sem se preocupar em atribuir identidade aos veículos.

Vale ressaltar aqui que os dados necessários para a constituição de uma simulação que esteja alinhada ao padrão de coleta de dados da CET-SP são agregados e apontam para o uso de simuladores macroscópicos ou mesoscópicos, modelos que necessitam de muito menos esforços para calibragem do que modelos microscópicos [Burghout, 2004]. Estes dados são: o fluxo de saturação dos segmentos de rua no seu ponto de saída, que é usado para reger a vazão de cada link da simulação; o tempo de percurso livre médio de cada segmento, que rege a velocidade com que os carros percorrem cada link; o fluxo, ou contagem, que diz a frequência com a qual passam carros no segmento; e as probabilidades de conversão, que regem o perfil de saída dos carros de cada segmento. Estes dados serão importantes para que o ambiente simulado represente de forma verossímil o ritmo de vazão, o crescimento, propagação e dissipação das filas e os perfis de envio e chegada de carros entre um semáforo e outro.

Um dos fatores mais importantes a serem simulados, principalmente em redes congestionadas é a propagação das filas, mais especificamente o cálculo do ponto efetivo de bloqueio, o fim delas. Neste modelo, este cálculo é feito de forma explícita a partir do perfil de chegada dos carros: para calcular o crescimento da fila, a cada passo de simulação os carros que estão empilhados em um link são contados e um comprimento inicial da fila é calculado, em seguida cada carro que se encontra em movimento mas que esteja à frente deste fim da fila é incrementado; para simular a propagação da fila, foi implementado conceito de propagação de espaços, que representa o fato de que ao sair do segmento pela linha de retenção, todo carro deixa um espaço igual na fila, que vai se propagando para trás, isso remete à ideia complementar de semáforos móveis que representam os momentos em que os carros podem ou não andar; por último, para representar a dissipação das filas estes semáforos, quando verdes, se chegam ao fim da fila, são eliminados para retroceder este ponto de bloqueio.

A velocidade de propagação destes espaços respeita em sua formulação o fluxo de saturação e o tempo de percurso da via para cada link e este modelo permite, principalmente, que seja simulado um atraso realista entre a liberação de carros no ponto de saída de um link lotado, por exemplo, e o momento em que o link à montante pode inserir carros nele. Este seria um exemplo básico, mas essa descrição também tem por consequência a observação de todos os efeitos de propagação de filas compostos pelos 
movimentos de crescimento e dissipação, independente do perfil e ritmo de chegada e saída dos carros. Assim, é permitido ao usuário contemplar os diversos pelotões que compõem uma única fila no caso dessa ser bem extensa (Figura 2).

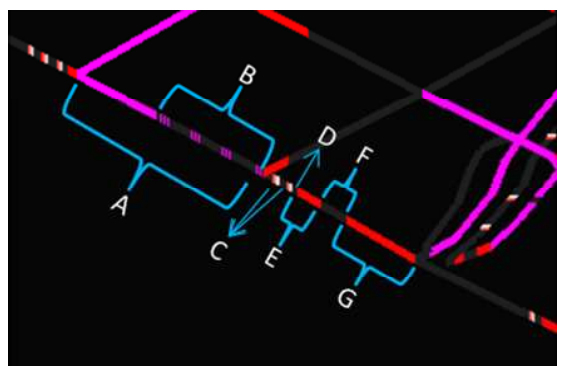

A- Link Bloqueado (fim da fila no final do segmento);

B e F- Espaço se Propagando;

C- Carros em Movimento;

D- Fim da última Fila do segmento;

E- Segunda Sub-Fila; G- Primeira SubFila

\section{Figura 2. Parte de uma simulação mostrando algumas vias congestionadas}

Sobre esse modelo de simulação é aplicado um AG em que, para cada indivíduo testado (cada plano semafórico) existe um cromossomo com duas partes que representam as defasagens entre os semáforos e os graus de saturação de cada aproximação de um semáforo. Como os graus de saturação recomendados [Vilanova, 2005a] situam-se entre $80 \%$ e $90 \%$, optou-se por trabalhar com 16 níveis de discretização (4 bits) entre $76 \%$ e $91 \%$. Enquanto isso, o tempo de ciclo máximo adotado para o estudo de caso foi o de 120 segundos e optou-se trabalhar com -64 a 63 segundos de defasagem (7 bits).

O tipo de seleção escolhido foi baseado em janelamento onde os mais fortes serão mais favorecidos, e para que se aumente a pressão evolutiva e se permita rápidas convergências. Existe alguma dúvida se isso pode levar a convergências prematuras, de modo que outros métodos de seleção serão testados no futuro. Cada dois indivíduos selecionados geram dois filhos diferentes que recebem mutações binárias com probabilidade de $1 \%$ para cada bit. A técnica de cruzamento escolhida foi a de corte duplo semelhante ao corte circular, onde ao filho é atribuída uma metade de cada cromossomo pai, sendo que o ponto de corte inicial será definido aleatoriamente para cada cruzamento entre o início e a metade do cromossomo. No estudo de caso o índice de mérito escolhido é composto pelo atraso total somado ao número de paradas seguindo a fórmula $\mathrm{Fit}=\mathrm{A}+20^{*} \mathrm{P}$. A primeira componente reflete efetivamente quanto tempo é desperdiçado pelos motoristas na rede toda, enquanto que a segunda componente reflete o custo ambiental e psicológico das sucessivas frenagens $\mathrm{e}$ arrancadas que se dão ao longo do percurso.

No programa foi aplicado o gerador de números aleatórios "Mersenne Twister" [Matsumoto e Nishimura, 1998] para todos os elementos que requeriram números aleatórios, da inserção de carros pelos links de entrada e sorteio de suas conversões ao longo da simulação, à geração das populações iniciais de cromossomos, e estabelecimento de pontos de corte para cruzamentos e as mutações.

\section{Estudo de caso}

Durante o projeto foi mantido contato com a CET-SP e, após a programação da ferramenta, foi recebida uma sub-rede da zona sul de São Paulo, no bairro do Brooklin, para estudo de caso. A rede (Figura 3) é composta por 6 semáforos de duas fases e 
compreende a região descrita pela rua Padre Antonio José dos Santos e Rua Indiana, com as travessas desde a Rua Portugal até Rua Guaraiuva.

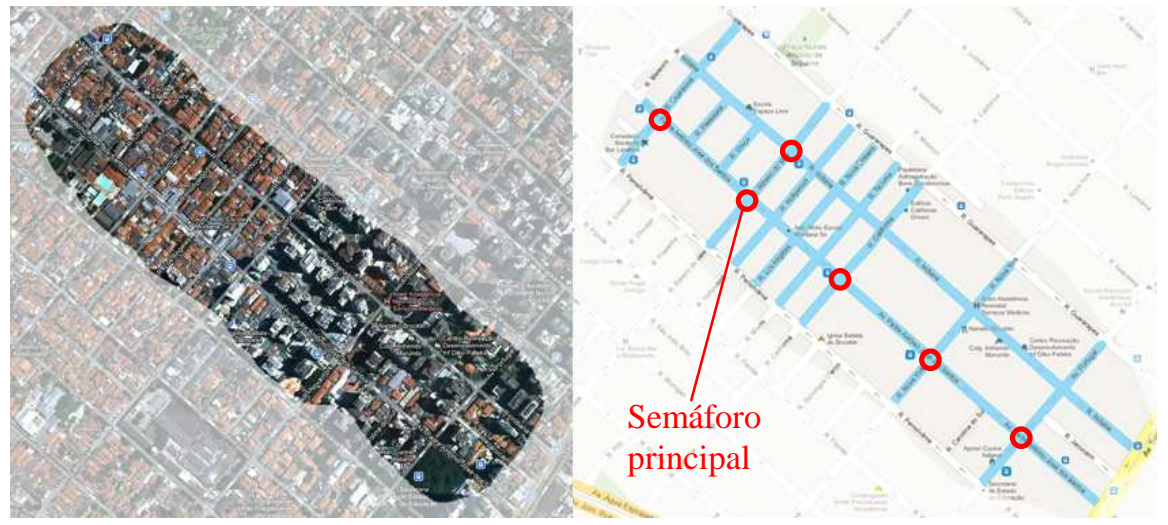

Figura 3. Mapa do estudo de caso

A coleta de dados foi realizada por uma única pessoa, de modo que no caso dos fluxos de saturação e dos tempos de percurso foi necessário realizar algumas compactações para agilizar a parametrização. Agrupamentos de segmentos que possuem as mesmas conformidades topológicas, como condição da pavimentação, inclinação, sinalização de via preferencial ou não e valetas, receberam parâmetros iguais sejam eles apenas o fluxo de saturação, o tempo de percurso, ou ambos.

As contagens de fluxo e porcentagens de conversão foram realizadas ao longo de manhãs e tardes para otimizar os dois períodos de pico diários, um entre as 07:00 e 11:00 e o outro entre as 16:00 e 20:00. Devido à falta de contingente para a coleta de dados foi necessário realizar uma extrapolação para que toda a rede recebesse uma demanda dinâmica que se aproximasse aos perfis dos horários na região. Para isso, foi escolhido um cruzamento principal que para cada tarde ou manhã de leitura seria observado uma vez a cada duas janelas de 15 minutos, enquanto os outros cruzamentos seriam observados uma única vez nas outras janelas.

Com a repetição desse processo de medidas e através de ajustes baseados na demanda de pico do cruzamento principal foi possível obter um perfil aproximado de demanda para a rede e valores médios acerca do fluxo de todas as aproximações, perfil este que pode ser observado na figura 4.
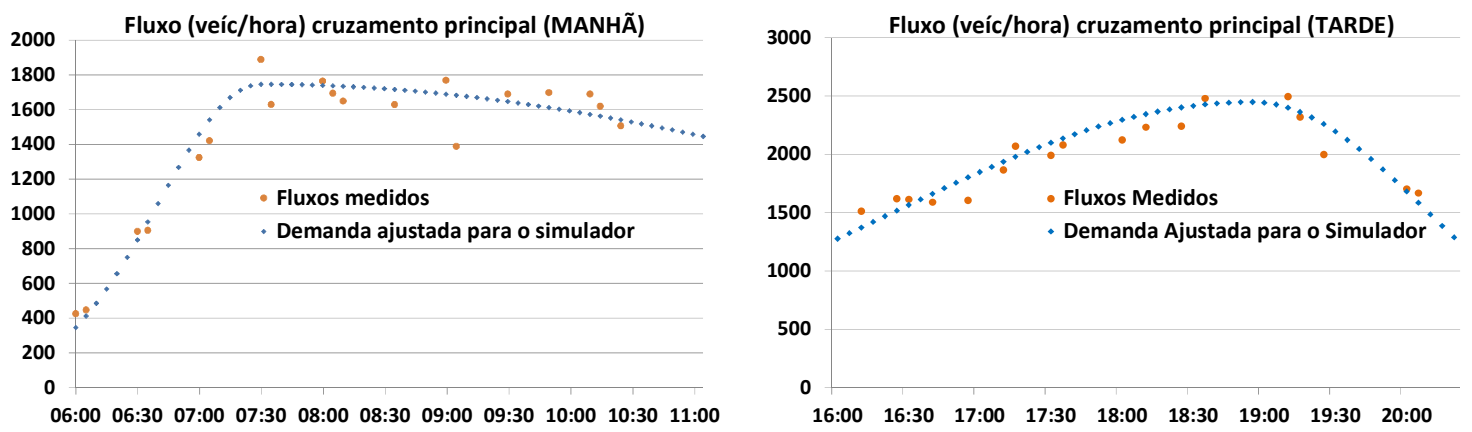

Figura 4. Perfis de demanda dos picos da manhã e da tarde respectivamente

Após a coleta de dados, foi feita a parametrização manual da rede para cada período de pico, sendo que para cada situação algumas otimizações foram feitas e seus 
planos de temporização registrados. No caso da manhã, não foi identificada saturação da rede, o que permitiu realizar otimizações com alguns níveis de demanda, de $100 \%$ em relação às leituras até a $130 \%$, ponto onde a rede apresentou saturação e os planos otimizados atingiram o tempo de ciclo máximo permitido (120 segundos).

Seguindo as recomendações encontradas no manual do software Transyt-7F [Hale, 2008], o tamanho da população do AG executado foi de 20 indivíduos e o número de gerações escolhido foi 200 . Os resultados encontram-se na figura 5.
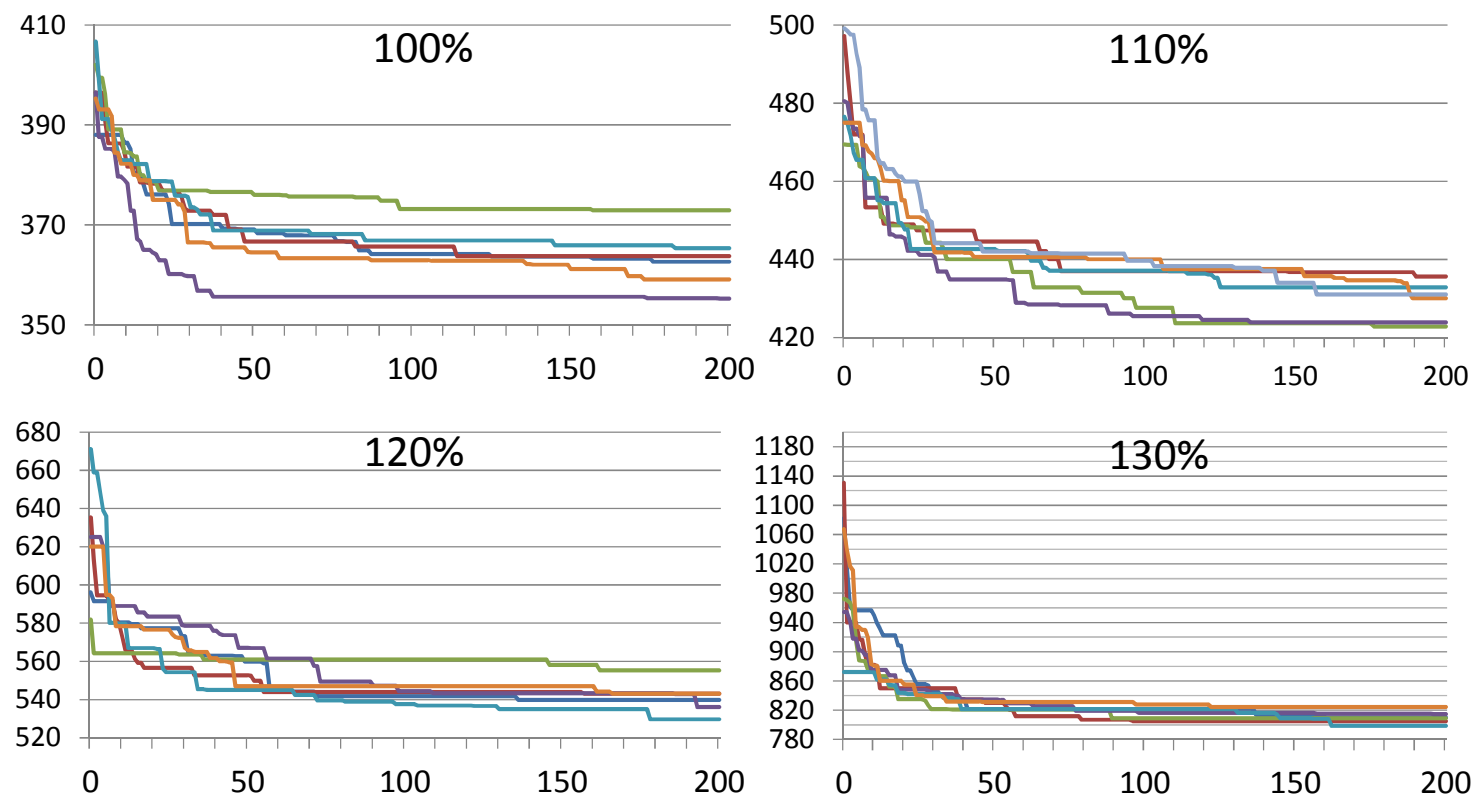

Figura 5. Curvas de evoluçao do melhor fitness de cada geração em função do número de gerações. Foram realizadas seis otimizações (cores distintas) para cada nível de demanda (de 100 a $130 \%$ do esperado), cada qual com uma semente de pseudo-aleatoriedade distinta.

Assim foi possível criar uma tabela (Figura 6) que comparasse, frente aos diferentes níveis de demanda, os desempenhos simulados dos planos otimizados para cada cenário e do plano anterior. Com a tabela foi possível escolher o plano mais robusto, que foi implantado na rede real.

\begin{tabular}{|c|c|c|c|c|c|c|c|c|c|c|c|c|}
\hline & \multicolumn{4}{|c|}{ Atraso } & \multicolumn{4}{|c|}{$20 *$ Paradas } & \multicolumn{4}{|c|}{ Fitness $(\mathbf{A}+20 * \mathbf{P})$} \\
\hline Demanda & $100 \%$ & $110 \%$ & $120 \%$ & $130 \%$ & $100 \%$ & $110 \%$ & $120 \%$ & $130 \%$ & $100 \%$ & $110 \%$ & $120 \%$ & $130 \%$ \\
\hline $100 \%[56]$ & 139 & 188 & 439 & 1228 & 222 & 257 & 367 & 657 & 361 & 445 & 806 & 1886 \\
\hline $110 \%[71]$ & 153 & 188 & 353 & 620 & 217 & 243 & 304 & 378 & 371 & 430 & 657 & 997 \\
\hline $\mathrm{PA}[$ 72] & 208 & 379 & 1521 & 3179 & 223 & 291 & 633 & 1114 & 432 & 670 & 2153 & 4294 \\
\hline$\frac{10}{2} 120 \%[90]$ & 197 & 231 & 301 & 627 & 216 & 239 & 271 & 362 & 413 & 470 & 572 & 989 \\
\hline $130 \%$ [111] & 252 & 293 & 375 & 500 & 217 & 239 & 268 & 312 & 469 & 532 & 625 & 813 \\
\hline
\end{tabular}

Figura 6. Desempenhos de planos otimizados e do anterior para pico da manhã

Para o pico da tarde foi identificada saturação da rede durante as leituras, então somente se otimizou o nível de demanda aferido, sendo escolhido o plano otimizado que possuia maior quantidade de semelhanças com os demais. Na figura 7 há uma síntese com a curva de evolução do fitness, a tabela comparativa entre os desempenhos do plano otimizado aplicado e do anterior e as temporizações dos semáforos para cada um. 
Os planos otimizados encontram-se aplicados na rede real e, analisados por engenheiros da CET-SP, já apresentaram bons resultados em casos isolados de demanda atipicamente alta devido a redirecionamentos de tráfego causados por ocorrências em redes próximas, mas leituras mais conclusivas ainda precisam ser feitas, pois a implantação foi feita no período de férias escolares e portanto a demanda média diária encontra-se abaixo do esperado.

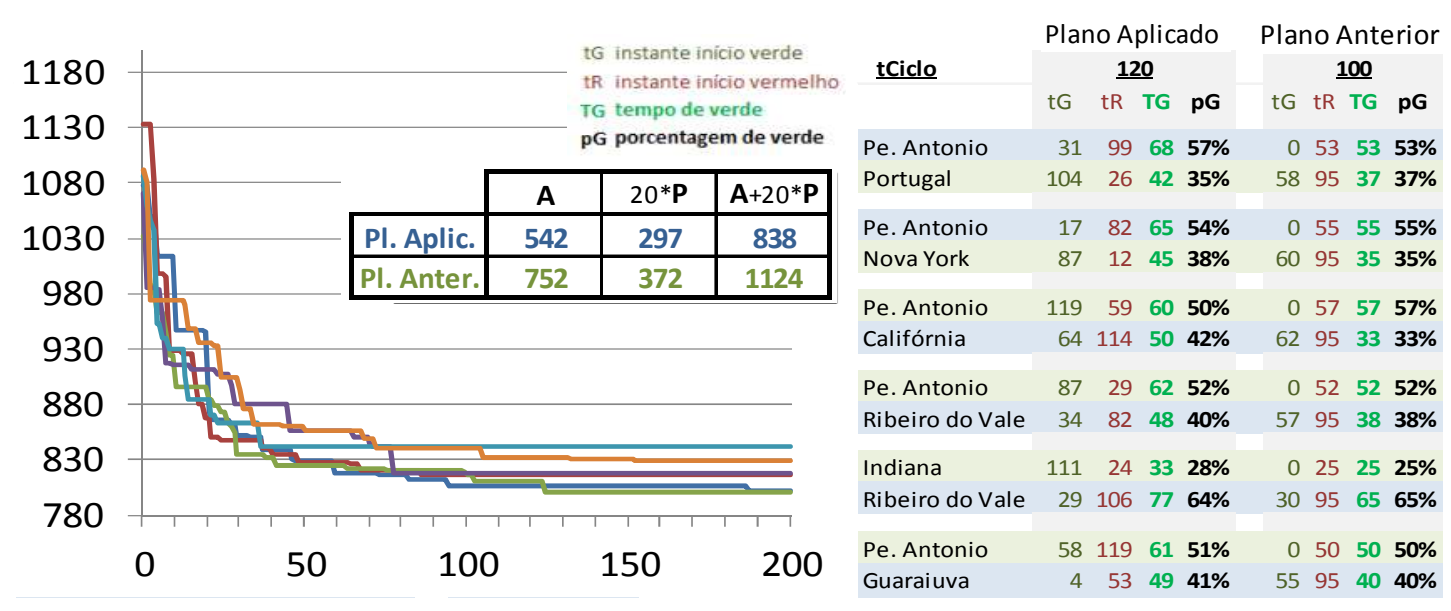

Figura 7. Otimização para o pico da tarde, plano semafórico encontrado e tabelas comparativas entre temporização do plano aplicado e do anterior

Em relação ao tempo de processamento, cada simulação de um período de 4 horas demora de 0,5 a 0,7 segundos no computador usado, provido de processador Intel Core i5 de $2.4 \mathrm{GHz}$ e 4 GB de memória RAM DDR3 $1333 \mathrm{MHz}$, dependendo do quanto a rede estiver carregada. Isso implica em tempos de otimização de cerca de 40 minutos para os valores escolhidos de tamanho da população e números de gerações (4000 simulações). Esse número pode ser multiplicado se for escolhido realizar mais de uma otimização por cromossomo para obter valores de fitness médios, o que foi a opção neste estudo de caso para as simulações de situações mais congestionadas (3 ou 4 repetições) devido à grande volatilidade apresentada, semelhante a redes reais.

\section{Conclusões}

Como esperado, a ferramenta desenvolvida obteve resultados simulados mais expressivos no caso das redes congestionadas e se apresentou como boa alternativa para obtenção de soluções para temporização de semáforos. Apesar da situação de escassez de dados, e das consequentes aproximações e extrapolações das leituras, os resultados da aplicação real trouxeram bons indícios para que o desenvolvimento de um simulador mais avançado, juntamente à implementação de técnicas de AGs mais elaboradas e com melhores desempenhos, tragam resultados melhores e mais próximos a realidade do que a versão atual. Além disso, no futuro seria interessante realizar o mesmo estudo, mas dispondo de maior contingente para a realização das leituras para a obtenção de dados mais precisos que permitam os traçados dos perfis de demanda dinâmico para cada aproximação ao invés de apenas um cruzamento principal.

Outro resultado interessante alcançado na elaboração do modelo foi o relativo à velocidade da simulação, que, mesmo sem qualquer otimização na codifiação, é comparável àquela da aplicação online no trabalho de Pohlmann e Friedrich (2011), 
indicando que a arquitetura aqui desenvovida favoreceria AGs para a otimização de planos semafóricos em tempo real mesmo sem o uso de computação paralela, que também é uma ótima alternativa para a viabilização desse tipo de aplicação.

\section{Referências}

Brownlee, J. (2011) Clever Algorithms - Nature Inspired Programming Recipes, Creative Commons Attribution-Noncommercial-Share Alike 2.5 Australia License, livro de referências, $436 \mathrm{p}$

Cantarella, G.E. e Pavone, G.; Vitetta, A. (2006) Heuristics for urban road network design: Lane layout and signal settings, European Journal of Operational Research Volume 175, Issue 3, artigo, 14p

Cavicchio, D. (1972) Reproductive Adaptive Plans, Proceedings of the ACM annual conference-Volume 1, artigo, 11p

Ceylan, H. e Bell, Michael G. H. (2004a) Reserve Capacity for a Road Network Under Optimized Fixed Time Traffic Signal Control, Journal of Intelligent Transportation Systems Volume 8, Issue 2, artigo, 14p

Ceylan, H. e Bell, M. G. H. (2004b) Traffic signal timing optimisation based on genetic algorithm approach, ncluding drivers routing, Transportation Research Part B: Methodological Volume 38, Issue 4, artigo, 14p

Ejzenberg, S. (2005) Reprogramação de Semáforos - Método de Observação de Campo, website instrucional Sinal de Trânsito (http://www.sinaldetransito.com.br) - CET$\mathrm{SP}$, artigo, $32 \mathrm{p}$

Hale, D. K. (2008) TRAffic Network StudY Tool, TRANSYT-7F, United States Version, McTrans Center - University of Florida, manual, 442p

Holland, J. H. (1992) Genetic Algorithms, http://econ2.econ.iastate.edu/tesfatsi/holland.gaintro.htm

Kesur, K. B. (2009), Advances in Genetic Algorithm Optimization of Traffic Signals, Journal of Transportation Engineering 135, Issue 4, artigo, 14p

Lebdeh, G. A. e Benekohal, R. (1997), Development of Traffic Control and Queue Management Procedures for Oversaturated Arterials, Transportation Research Record: Journal of the Transportation Research Board, Volume 1603, artigo, 9p

Lo, H. K, Chang E. e Chan, Yiu C. (2001) Dynamic Network traffic control, Transportation Research Part A: Policy and Practice, Volume 35, Issue 8, artigo, 24p

Lopes, D.L. (2003), Viabilidade do Uso de Modelos Sintéticos Integrados de Uso de Solo e Transportes: Estudo de Aplicação à Cidade de São Paulo, Escola Politécnica da USP, tese mestrado, $177 \mathrm{p}$ 
Medina, J. J. S., Moreno, M. J. G. e Royo, E. R. (2010) Traffic Signal Optimization in "La Almozara" District in Saragossa Under Congestion Conditions, Using Genetic Algorithms, Traffic Microsimulation, and Cluster Computing, IEEE Transactions on Intelligent Transportation Systems, Volume 11, Issue 1, artigo 10p

Mitchell, M. (1995) Genetic algorithms: An overview, Santa Fe Institute, artigo, 17p

Parl, B., Messer, C. e Urbanik, T. (2000) Enhanced Genetic Algorithm for SignalTiming Optimization of Oversaturated Intersections, Transportation Research Record: Journal of the Transportation Research Board, Volume 1727, artigo, 10p

Park, B., Santra, P., Yun, I. e Lee D. H. (2004) Optimization of Time-of-Day Breakpoints for Better Traffic Signal Control, Transportation Research Record: Journal of the Transportation Research Board, Volume 1867, artigo, 7p

Pohlmann, T. e Friedrich, B. (2010) A new online control strategy for signalized urban sub-networks, 12th World Congress on Transportation Research, artigo, 22p

Rennard, J. P. (2000), Introduction do Genetic Algorithms (http://www.rennard.org/alife/english/gavintrgb.html), website educativo

Robertson, D. I. e Bretherton, R. D. (1991) Optimizing Networks of Traffic Signals in Real Time-The SCOOT Method, IEEE Transactions on Vehicular Technology, Volume 40, Issue 1, artigo, 5p

Sun D; Benekohal, R. F. e Waller T. (2006) Bi-level Programming Formulation and Heuristic Solution Approach for Dynamic Traffic Signal Optimization, ComputerAided Civil and Infrastructure Engineering, Volume 21, Issue 5, artigo, 13p

Stevanovic, A., Martin P., e Stevanovic J. (2007) VisSim-Based Genetic Algorithm Optimization of Signal Timings, Transportation Research Record: Journal of the Transportation Research Board, Volume 2035, artigo, 10p

Teklu, F.; Sumalee, A.; Watling, D. (2007), A Genetic Algorithm Approach for Optimizing Traffic Control Signals Considering Routing, Computer-Aided Civil and Infrastructure Engineering, Volume 22, Issue 1, artigo, 13p

Vilanova, L. (2005a) Programação de um semáforo usando o método do grau de saturação, website instrucional Sinal de Trânsito (http://www.sinaldetransito.com.br), artigo, $14 \mathrm{p}$

Vilanova, L. (2005b) Fundamentos da programação semafórica, website instrucional Sinal de Trânsito (http://www.sinaldetransito.com.br) - CET-SP, artigo, 20p 